Forschende

Komplementärmedizin

Wissenschaft • Praxis • Perspektiven

\section{Acknowledgement to Reviewers - Dank an Gutachter}

Forsch Komplementmed 2014;21:347

The editors extend their gratitude and appreciation to the following reviewers whose comments and criticism ensure the quality of the articles published in this journal.

Terje Alræk, Troms $\varnothing$

Hakima Amri, Washington D.C.

Marcus Bäcker, Düsseldorf

Stephan Baumgartner, Arlesheim

Simon Becker, Horgen

Kathrin Bernardy, Bochum

Rainer Brenke, Berlin

Arndt Büssing, Herdecke

Kate Chatfield, Preston

Holger Cramer, Essen

Wolfgang Doerfler, München

Axel Eustachi, München

Kerstin Finger, Templin

Lorenz Fischer, Bern

Johannes Fleckenstein, München

Michael Frass, Wien

Bruno Frank, Kleinrinderfeld

Christopher J. Fries, Winnipeg, MB

Rainer Görne, Neustadt an der

Weinstraße

Kathrin Gschwendtner, Freiburg i.Br.

Corina Güthlin, Frankfurt/M.

Harald J. Hamre, Freiburg i.Br.

Thomas Heidenreich, Esslingen

Hartmut Heine, Neuhausen

Andreas Hensel, Münster

Roman Huber, Freiburg i.Br.

Josef Hummelsberger, München

Hubert Janik, Rostock

Jean Pierre Jansen, Groningen

Helle Johannessen, Odense

Uwe Juergens, Bonn

Reichling Jürgen, Heidelberg

Markus Keller, Langgöns

Romy Lauche, Essen

Klaus Linde, München

Liu, Jianping, Troms $\varnothing /$ Beijing
Harald Matthes, Berlin

Jörg Melzer, Zürich/Berlin

Matthias F. Melzig, Berlin

Susanne Moebus, Essen

Heribert Möllinger, Lugano

Albrecht Molsberger, Düsseldorf

Frauke Musial, Troms $\varnothing$

Karen Nieber, Leipzig

Miriam Ortiz, Berlin

Thomas Ostermann, Herdecke

Florian Pfab, Charlestown

Roja Rahimi, Tehran

HeidrunReißenweber-Hewel,

Gräfelfing

Karl-Ludwig Resch, Bad Elster

Paolo Roberti di Sarsina, Bologna

Marc Schläppi, St. Gallen

Stefan Schmidt, Freiburg i.Br.

Ulrich Schwantes, Oberkrämer

Florian Schwerla, Gauting

Andreas Sönnichsen, Herdecke

Rainer Stange, Berlin

Wolfram Stör, Icking

Fiona Streckmann, Köln

Tine Strub, Troms $\varnothing$

Saha Subrhanil, New Delhi

Michael Teut, Berlin

Bernhard Uehleke, Berlin

Harryvan der Zee, Haren

Petra Voiss, Essen

Sabine Vollstedt, Bokholt-Hanredder

Klaus von Ammon, Bern

Cornelia U. von Hagens, Heidelberg

Michael Walkenhorst, Frick

Stefan W. Weinschenk, Karlsruhe

Axel Wiebrecht, Berlin

$\mathrm{Xu}$ Zhang, Nanjing

\title{
KARGER
}

(c) 2014 S. Karger GmbH, Freiburg 